\title{
Genome Editing of Rice PFT1 Gene to Study its Role in Rice Sheath Blight Disease Resistance
}

\author{
Priya Rajesh Shah ${ }^{1}$, S. Varanavasiappan ${ }^{1}$, E. Kokiladevi ${ }^{1}$, \\ A. Ramanathan ${ }^{2}$ and K.K. Kumar ${ }^{*}$
}

${ }^{1}$ Department of Plant Biotechnology, Tamil Nadu Agricultural University, Coimbatore, India

${ }^{2}$ Tamil Nadu Rice Research Institute, Aduthurai, Thanjavur, Tamilnadu, India

*Corresponding author

\section{Keywords}

Gene editing, CRISPR/Cas9, Rice transformation, Phytochrome and flowering time 1 , Sheath blight resistance

Article Info

Accepted:

17 May 2019

Available Online:

10 June 2019

\section{A B S T R A C T}

The CRISPR/Cas9 system has been used predominantly for precise editing of the plant genomes. In the present study, we have made an application of CRISPR/Cas9 system for targeted mutagenesis in rice(Oryza sativa L.) targeting the Phytochrome and Flowering Time 1 ( $P F T 1$ ) gene known to be involved in disease susceptibility in Arabidopsis for rootinfecting hemibiotrophic fungal pathogen Fusarium oxysporum. Hence, to confirm the role of OSPFT1 gene in rice sheath blight disease, CRISPR-Cas9 gene editing tool is being employed for generating OsPFTl gene knock out in rice. Two guide RNAs (gRNAs) were designed to pair with distinct $O S P F T 1$ gene region followed by protospacer-adjacent motif (PAM). Rice genome editing constructs were cloned and mobilized into the Agrobacterium strain LBA4404. The transgene (Cas9-gRNA) introduction into indica rice variety ASD16was done via Agrobacterium-mediated rice transformation by using immature embryos as explants. The gRNA will direct the Cas9 nuclease to generate double-strand breaks (DSB) at the specific sites of OsPFT1 gene, thereby introducing mutations at the DSB by error-prone non-homologues end joining repair mechanism. Through PCR analysis, the presence of the hpt transgene was identified. Homozygous gene-edited transgenic rice plants will be identified and further will be subjected to sheath blight disease screening.

\section{Introduction}

Genome targeted mutagenesis plays a crucial role in crop improvement. CRISPR/Cas9 has been an emerging molecular tool in the field of genome editing (Sun et al., 2016). It is a powerful RNA-guided DNA targeting platform for editing the genome of different organisms (Jiang and Doudna 2017). This technology makes use of the specific $\sim 20 \mathrm{bp}$ guide RNA for targeting the specific genomic loci (Ran et al., 2013). Rice (Oryza sativa L.) is susceptible to many diseases including sheath blight. Rice sheath blight (ShB) is caused by Rhizoctonia solani Kuhn, [Teleomorph stage, Thanatephorus cucumeris (Frank) Donk]. It is regarded as a second internationally important disease after rice blast (Dasgupta, 1992). Yield loss of $8 \%$ to $50 \%$ was reported due to ShB (Savary et al., 
2000). The study made by Backstrom et al., (2007) has suggested that PFT1 protein (MEDIATOR25) is a part of the conserved multiprotein mediator complex that plays an essential role in initiating transcription by acting as a "universal adaptor" between RNA polymerase II and DNA-bound transcription factors. Arabidopsis PFTl gene is involved in disease susceptibility to root-infecting hemibiotrophic fungal pathogen Fusarium oxysporum (Thatcher et al., 2009). A homolog of wheat of PFT1/MED25 complemented the defensive and developmental phenotypes of the $p f t 1$ mutant, which suggests about the conserved function of PFT1 in wheat and possibly in rice as well (Kidd et al., 2009). Hence, an attempt was made to characterize the functional role played by $O S P F T 1$ gene in rice ShB disease resistance and knocking out of OsPFTl by CRISPR-Cas9 to study its role in resistance.

\section{Materials and Methods}

\section{Designing candidate Cas9/gRNA target site in rice genome}

Targeted sites of OSPFTl were chosen (Fig. 1) in exon 1 and exon 3 and 20-nt unique guide sequences (5'A N20 GG 3') were identified by using the web based tool such as 'CRISPR PLANT' database (Xie et al., 2014) (http://www.genome.arizona.edu/crispr/) and CRISPR-P2.0 (Liu et al., 2017). The selected 20-nt sgRNA-binding sequence precedes immediately the NGG PAMto make the target sequence unique. The CRISPR-Cas9 based pRGEB32 vector was used for expressing the selected guide RNA. The pRGEB32 express Cas9 gene under rice ubiquitin promoter and possess hygromycin resistance gene (hpt) driven by CaMV35S promoter as plant selectable marker (Fig. 2). The PS-I and PS-II construct harbouring gRNA were prepared as follows: Firstly, the empty pRGEB32 vector was digested with $B s a \mathrm{I}$ and gel eluted. Then, oligo-duplex was prepared by using OsPFT1 gRNAs $\mathrm{F}$ and $\mathrm{R}$ sequence of IgRNA and IIgRNA respectively (Table 2). For ligation of the oligo-duplex with the appropriate adapters into the purified $B s a$ I digested vector, the oligo duplex was diluted 1:200 and separate ligation reaction was incubated at $4{ }^{\circ} \mathrm{C}$ overnight. The confirmation of ligated product was done by amplifying the ligated product using pRGEB32 specific forward and OsPFTlgRNA specific reverse primers or vice-versa (Table 2). The two PS-I and PS-II CRISPR-Cas9-gRNA constructs based on pRGEB32 harboring oligo duplex (gRNA) for knocking out OSPFT1 gene. The ligated products were then used to transform competent E. coli (DH5 $\alpha)$ cells. The constructs were further confirmed by restriction digestion by $B s a \mathrm{I}$ and $\mathrm{XbaI}$ of the plasmid DNA isolated from the transformed colonies followed by sequencing of the isolated plasmid DNA for PS-I and PS-II construct respectively.

\section{Transformation of Cas9 and gRNA constructs}

Agrobacterium-mediated transformation of indica rice cultivar ASD16using immature embryos derived calli was performed as described by Hiei and Komari (2008). Hence the PS-I and PS-II CRISPR/Cas9-gRNA constructs were mobilized into the Agrobacterium strain (LBA4404) by using triparental mating. Randomly isolated mobilized transconjugants colonies were selected for PCR screening for the presence of vir and hptII specific primer sets with appropriate PCR conditions. Then the isolated immature embryos were infected by Agrobacterium carrying the PS-I and PS-II constructs respectively. After 7 days of co-cultivation, the elongated shoots from immature embryos were removed and calli were transferred to resting medium, CCMC containing cefotaxim $250 \mathrm{mg} / \mathrm{l}$ to control Agrobacterium 
overgrowth. Further for the selection of transformed calli, the calli were placed on the selection medium, CCMCH50 supplemented with $50 \mathrm{~g} / \mathrm{l}$ hygromycin. Hygromycin resistant calli were selected over 17 days. Proliferating calli were then transferred to the regeneration media supplemented with $30 \mathrm{~g} / \mathrm{l}$ glutamine and $40 \mathrm{~g} / \mathrm{l}$ hygromycin. The regenerated shoots after 14 days were transferred to rooting medium followed by hardening in green house.

\section{Results and Discussion}

\section{Target selection and construction of the} CRISPR/Cas9 system

In rice (Oryza sativa), the PFT1 gene is located on chromosome 9 and the locus id is LOC_Os09g13610. Two sgRNAs (IgRNA and IIgRNA) targeting the first exon and third exon of PFT1 respectively were designed (Fig. 1) by the aid of web based tool 'CRISPR PLANT' database (http://www.genome. arizona.edu/crispr/) and CRISPR-P2.0. The designed sgRNA along with the various parameters are present in the Table 1.

The PS-I (pRGEB32 + IgRNA) and PS-II (pRGEB32 + IIgRNA) construct were prepared by ligating oligo duplex for IgRNA and IIgRNA with the appropriate adapters into the $B s a \mathrm{I}$ digested vector and were confirmed by the PCR analysis. The pRGEB32 vector specific forward and OsPFT1 gRNA specific reverse primer showed an expected amplification of 280bp while the other set of primer gRNA specific forward primer and $\mathrm{pRGEB} 32$ vector specific reverse primer (Table 2) shows specific amplification of $208 \mathrm{bp}$. The $B s a \mathrm{I}$ digested plasmid was used as negative control which does not show any amplification. The confirmed ligated products were transformed into the E. coli DH5a competent cells, the recombinant colonies were screened through colony PCR by the pRGEB32 vector specific forward and OsPFT1gRNA specific reverse primer (Table 2). The positive colonies were selected and subsequently the plasmid DNA was isolated from the recombinant colonies. Further confirmation was made by restriction digestion of the plasmid DNA by BsaI and $X b a \mathrm{I}$. The transformed colonies will not be digested by $B s a \mathrm{I}$ as due to the insertion of gRNA, the recognition site of $B s a \mathrm{I}$ was disturbed so there will be only single digestion by XbaI. Finally, PS-I and PS-II constructs were confirmed by sequencing.

CRISPR/Cas9- mediated genome editing of the PFT1 gene of indica rice for developing $\mathrm{ShB}$ resistance

Agrobacterium-mediated transformation of indica rice was done to knock out the PFTlgene using the CRISPR/Cas9 construct PS-I (pRGEB32+ IgRNA) and PS-II (pRGEB32 + IIgRNA), harboring OsPFT1 gene specific sgRNA and a hygromycin as plant selectable marker respectively. The gene constructs were mobilized into Agrobacterium strain LBA4404separately. The presence of the Cas9 plasmid, PS-I (pRGEB32 + IgRNA) and PS-II (pRGEB32 + IIgRNA) in the Agrobacterium strains were confirmed by colony PCR for the presence of 440bp virG, 686 bp hptII and integrity of construct was further confirmed by back transformation. Negative controls did not show any amplification in hptII/virG specific primers sets (Fig. 5). Total Agrobacterium genomic DNA was isolated from three PS-I (pRGEB32 + IgRNA) and PS-II (pRGEB32 + IIgRNA) transconjugant respectively followed by transformation in E. coli. Plasmid DNA was isolated from $E$. coli transformants and was used for restriction enzyme digestion. Restriction digestion of plasmid isolated from back-transformed E. coli for three PS-I (pRGEB32 + IgRNA) and PS-II (pRGEB32 + IIgRNA) respectively was done with $B s a \mathrm{I}$ and 
$X b a \mathrm{I}$ to confirm the presence of the OsPFT1 gene specific gRNA into the PS-I and PS-II constructs enzyme digestion resulted in only single digestion by $\mathrm{XbaI}$ as expected (Fig. 1-5).

Fig.1 Schematic map of the gRNA target site selection in the target OsPFTl gene. Two sgRNAs (I gRNA and II gRNA) of the PFTI gene were selected, corresponding to the sites in the first and the third exon, respectively

\section{GTACTGGCCCGTCACCGTGGCGG TTAGTCGTCTTCCATACCCATGG}

OsPFT1 gene

Chr-9:7914083 : 7925334

LOC_Os09g13610
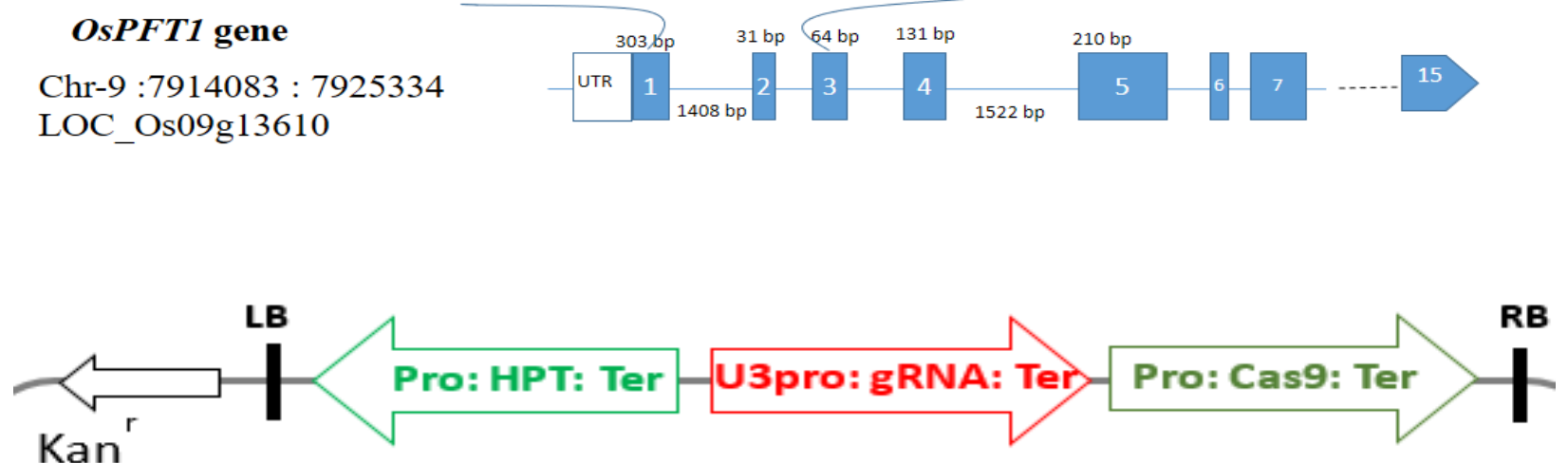

Kan

Fig. 2 CRISPR-Cas9-gRNA construct

Fig.3 PCR analysis of ligated products (a) I gRNA products with pRGEB32 F-primer and OsPFT1 R-primer (280 bp) and OsPFT1 F-primer and pRGEB32 R-primer (208 bp) (b) II gRNA products with pRGEB32 F-primer and OsPFT1 R-primer (280 bp) and OsPFT1 F-primer and pRGEB32 R-primer(208 bp) (Table 2) and (c) Restriction analysis of clones from the ligation of two dsOligo (I gRNA and II gRNA) fragments into BsaI digested pRGEB32 individually. The individual recombinant clones of PS-I construct (pRGEB $32+$ I gRNA) and PS-II (pRGEB $32+$ II gRNA) with $B s a \mathrm{I}$ and $X b a \mathrm{I}$. The $B s a \mathrm{I}$ undigested clones are the positive ones that are ready for mobilization into Agrobacterium.

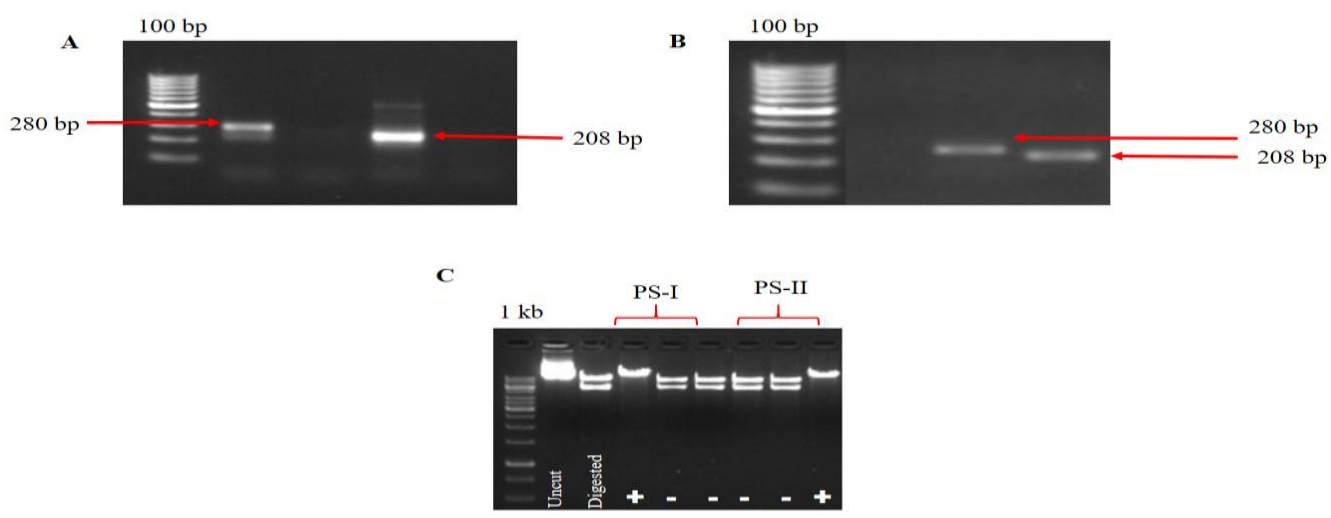


Fig.4 Sequence of the PS-I and PS-II cloned construct. Yellow label bars indicate the cloned I and II sgRNA sequence in the pRGEB32 vector

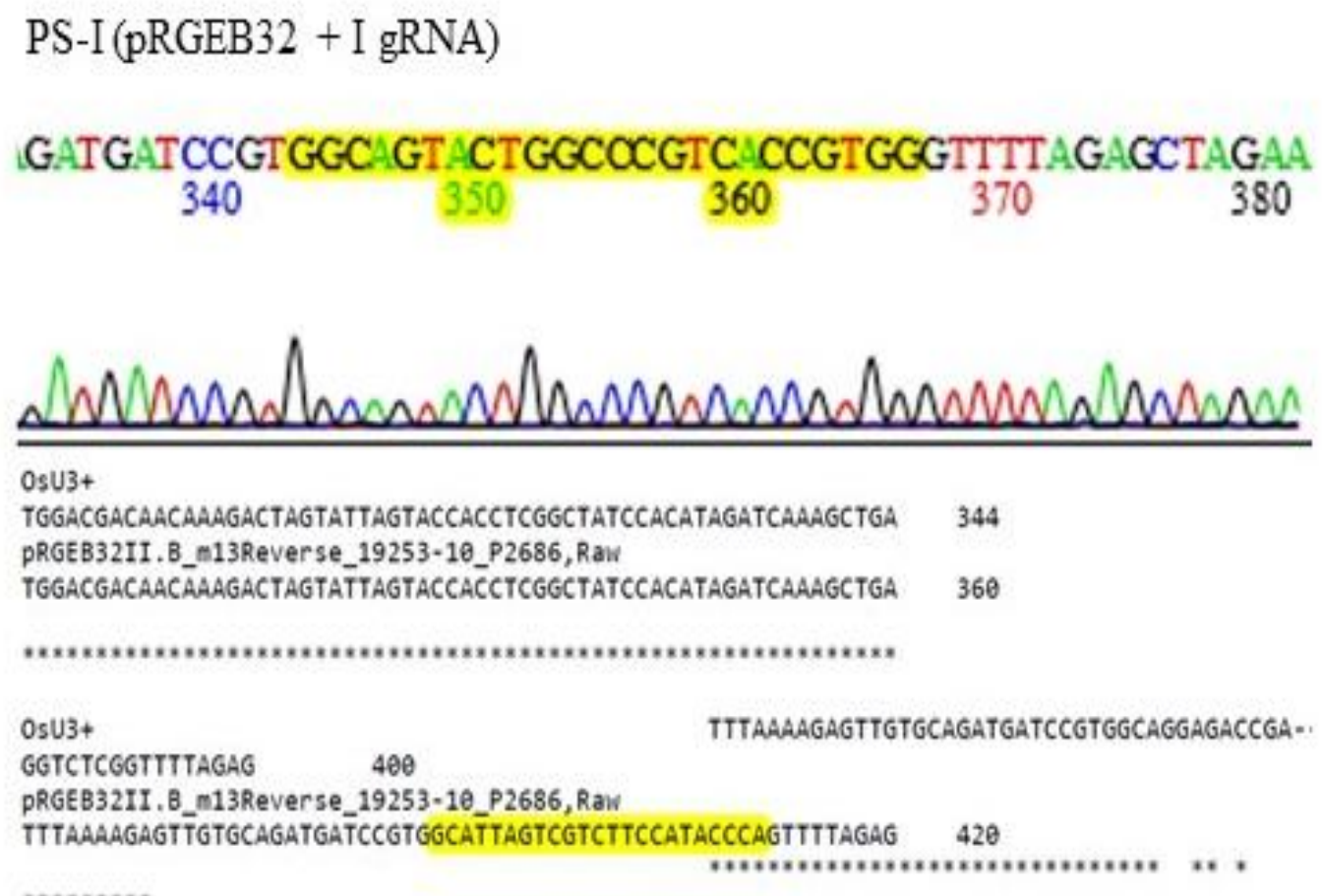

\section{PS-II (pRGEB32 + II gRNA)}
GAGTTGT GCAGATGATCCGTGGCATTAGTCGTCTTCCATACCCAGTTTTAGAGCTAGAAATAG $\begin{array}{llllll}290 & 300 & 310 & 320 & 330 & 340\end{array}$

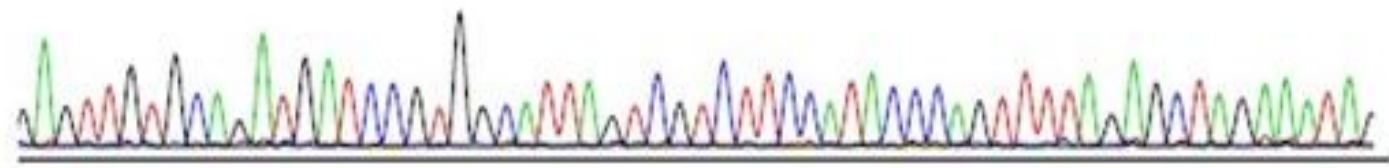

OsU3+

TGGACGACAACAAAGACTAGTATTAGTACCACCTCGGCTATCCACATAGATCAAAGCTGA 344

PRGEB3211.B_m13Reverse_19253-10_P2686, RaW

TGGACGACAACAAAGACTAGTATTAGTACCACCTCGGCTATCCACATAGATCAAAGCTGA 360

OSU3+

GGTCTCGGTTTTAGAG 460
PRGEB32II.B_m13Reverse_19253-10_P2686, Raw

TTTAAAAGAGTTGTGCAGATGATCCGTGGCATTAGTCOTCTTCCATACCCAGTTTTAGAG

TTTAAAAGAGTTGTGCAGATGATCCGTGGCAGGAGACCGA....

$* * * * * * * * *$ 
Fig.5 The clones of PS-I and PS-II construct were mobilized into Agrobacterium and PCR analysis of the TC (Transconjugants Colonies) by a and b virGand $\mathrm{c}$ and $\mathrm{d} h p t \mathrm{II}$ for PS-I and PS-

II construct respectively


Fig.6 Agrobacterium mediated transformation of immature embryos of rice cultivar ASD16



a. Rice immature embryos infected with Agrobacterium on NB-As medium

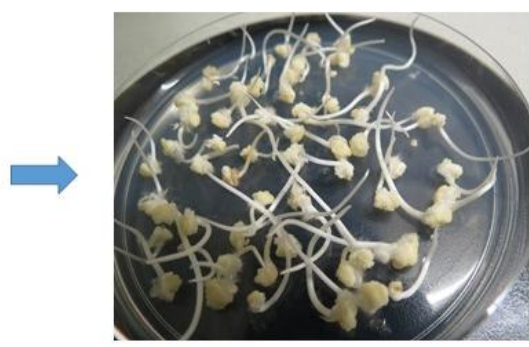

b. Immature embryos 7 days after co-cultivation in NB-As medium

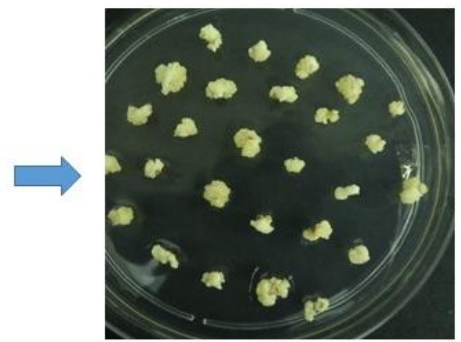

c. Calli sub-cultured on CCMC medium for proliferation



f. Rooting of regenerated calli on $1 / 2$ strength MS media

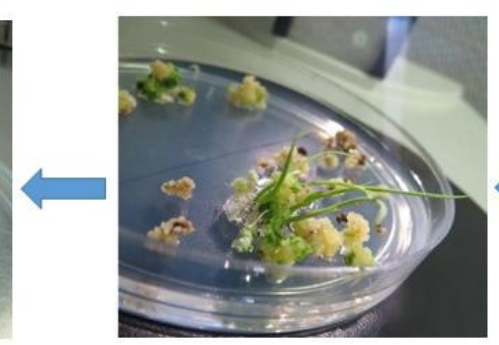

e. Regeneration of embryogenic calli on regeneration medium ( $\mathrm{RNMH} 30$ )



d. Calli on selection medium (CCMCH50) 
Table.1 Selected gRNAs on the basis of the various parameters

\begin{tabular}{|c|c|c|c|c|c|c|c|c|}
\hline $\begin{array}{l}\text { Sequence } \\
\text { ID }\end{array}$ & Spacer sequence (5' - 3') & PAM & $\begin{array}{l}\text { Str } \\
\text { and }\end{array}$ & $\begin{array}{l}\text { Locat } \\
\text { ion }\end{array}$ & $\begin{array}{l}\text { Exo } \\
\text { n } \\
\text { No. }\end{array}$ & $\begin{array}{l}\text { On } \\
\text { Targ } \\
\text { et }\end{array}$ & $\begin{array}{l}\text { Off } \\
\text { Tar } \\
\text { get }\end{array}$ & $\begin{array}{l}\text { Restriction } \\
\text { sites }\end{array}$ \\
\hline $\begin{array}{l}\text { Chr9:7914 } \\
340- \\
7914360\end{array}$ & $\begin{array}{l}\text { GTACTGGCCCGTCACC } \\
\text { GTGG }\end{array}$ & $\begin{array}{l}\text { CGGACT } \\
\text { ACGT }\end{array}$ & + & Exon & 1 & 0.61 & 22 & $\begin{array}{l}\text { HpyCH4IV } \\
\text { cut ACGT } \\
\text { EciI cut } \\
\text { GGCGGA }\end{array}$ \\
\hline $\begin{array}{l}\text { Chr9:7915 } \\
\text { 955- } \\
7915975\end{array}$ & $\begin{array}{l}\text { TTAGTCGTCTTCCATAC } \\
\text { CCA }\end{array}$ & $\begin{array}{l}\text { TGGTCCT } \\
\text { TAT }\end{array}$ & + & Exon & 3 & 0.75 & 19 & $\begin{array}{l}\text { NlaIII cut } \\
\text { CATG } \\
\text { CviAII cut } \\
\text { CATG } \\
\text { FatI cut CATG } \\
\text { NcoI cut } \\
\text { CCATGG }\end{array}$ \\
\hline
\end{tabular}

Table.2 Primer and sequences used in the protocol

\begin{tabular}{|c|c|c|}
\hline S. No. & Sequences (5'-3') & Purpose \\
\hline $\begin{array}{l}\text { pRGEB32- } \\
\text { F }\end{array}$ & AGGCGTCTTCTACTGGTGCT & $\begin{array}{l}\text { PCR for ligated product and clones } \\
\text { verification }\end{array}$ \\
\hline $\begin{array}{l}\text { pRGEB32- } \\
\text { R }\end{array}$ & CCTCCGTATTTGCTGACGTG & $\begin{array}{l}\text { PCR for ligated product and clones } \\
\text { verification }\end{array}$ \\
\hline $\begin{array}{l}\text { OsPFT1 g } \\
1 \mathrm{~F}\end{array}$ & $\begin{array}{l}\text { GGCAGTACTGGCCCGTCACCG } \\
\text { TGG }\end{array}$ & Cloning sgRNA into pRGEB32 \\
\hline $\begin{array}{l}\text { OsPFT1 g } \\
1 \mathrm{R}\end{array}$ & $\begin{array}{l}\text { CATGACCGGGCAGTGGCACCC } \\
\text { AAA }\end{array}$ & $\begin{array}{l}\text { PCR for ligated product and clone verification } \\
\text { Cloning sgRNA into pRGEB } 32\end{array}$ \\
\hline $\begin{array}{l}\text { OsPFT1 g } \\
2 \text { F }\end{array}$ & $\begin{array}{l}\text { GGCATTAGTCGTCTTCCATACC } \\
\text { CA }\end{array}$ & $\begin{array}{l}\text { PCR for ligated product and clone verification } \\
\text { Cloning sgRNA into pRGEB32 }\end{array}$ \\
\hline $\begin{array}{l}\text { OsPFT1 g } \\
2 \text { R }\end{array}$ & $\begin{array}{l}\text { AATCAGCAGAAGGTATGGGTC } \\
\text { AAA }\end{array}$ & $\begin{array}{l}\text { PCR for ligated product and clone verification } \\
\text { Cloning sgRNA into pRGEB32 }\end{array}$ \\
\hline & & PCR for ligated product and clone verification \\
\hline
\end{tabular}

The protocol reported by Hiei and Komari (2008), was used for rice transformation. Immature embryos of ASD16 rice plants were used as explants in Agrobacterium-mediated transformation experiments with PS-I and PS-
II gene construct. After co-cultivation, the developed embryogenic calli were subcultured twice on hygromycin containing selection medium. The hygromycin resistant calli survived selection process and grew well 
in two rounds of selection, but the untransformed calli turned brown and dried. The calli lines of ASD16 were recovered after two rounds of hygromycin selection. The selected embryogenic calli obtained after two rounds of selection were transferred to preregeneration and then to regeneration medium for shoot induction.

On regeneration medium, calli exhibited greening and small shoots started emerging within 8-10 days (Fig. 6). The transgenic plants obtained will then be subjected to the target mutation analysis by PCR/RE assay(Shan et al., 2014, Char et al., 2017). In PCR/RE assay, target locus includes restriction enzyme site that is destroyed by CRISPR/Cas-induced mutation. The CRISPR/Cas-PFT1 mutant will remain undigested while the non-mutant shows the digested bands. Further characterization of uncleaved bands can be made by sequencing. So as to validate these transgenic plants further molecular analysis has to be done by the PCR/RE assay as well as by sequencing to observe the expected mutation in the regenerated plant. In addition, the performance against the sheath blight disease will be assessed in the transgenic plants mutated for OsPFT1.

CRISPR/Cas9 has proven to be widely emerging and applicable genome editing tool for the production of new improved varieties, which exhibits enhanced disease resistance and other improved traits such as stress tolerance, nutritional improvement, and yield increment (Zong et al., 2017). The production of rice is hampered by various biotic and abiotic constraints among these the fungal, bacterial and viral constraints are the major ones. One such fungal constraint is rice sheath blight (ShB) and the causal agent for it is Rhizoctonia solani. For control of ShB in rice via biotechnological approaches, an attempt was made for knocking out of the susceptibility gene PFT1 (Kidd et al., 2009). The $20 \mathrm{bp}$ guide RNAs were designed for targeting the initial exon that is the exon 1 and 3 so that initially there is no expression of PFT1gene.A ShB susceptible indica rice cultivar, ASD16 was selected for the transformation with PS-I and PS-II CRISPR/Cas9 construct mobilized into Agrobacterium. In this study, immature embryos were used as target tissue for transformation since the immature embryo has high regeneration and transformation efficiency, although both immature embryos and mature seeds have been reported to be an excellent starting material for rice transformation via Agrobacterium method (Hiei and Komari 2008). After several rounds of Agrobacterium-mediated transformation, putative transgenic plants were recovered. Transgenic plants generated will be tested for its performance against the sheath blight disease.

In conclusions, rice (Oryza sativa L.) is one of the most widely cultivated, at the same time affected by various biotic and abiotic factors. Rice trait improvement appears really promising for the future and will surely be influenced by the developments in CRISPR/Cas9 technologies. This induced mutation will help to generate disease resistant rice varieties. In our paper, we efficiently applied targeted mutagenesis in immature embryos rice cultivar ASD16 using the CRISPR/Cas9 system and transgenic rice plants were generated. The CRISPR/Cas9 system can be used for targeted mutations and facilitating rice genetic improvement.

\section{References}

Bäckström, Stefan, Nils Elfving, Robert Nilsson, Gunnar Wingsle, and Stefan Björklund. 2007. "Purification of a plant mediator from Arabidopsis thaliana identifies PFT1 as the Med25 subunit." Molecular cell 26 (5):717729. 
Char, Si Nian, Anjanasree K Neelakandan, Hartinio Nahampun, Bronwyn Frame, Marcy Main, Martin H Spalding, Philip W Becraft, Blake C Meyers, Virginia Walbot, and Kan Wang. 2017. "An Agrobacterium- delivered CRISPR/Cas9 system for high- frequency targeted mutagenesis in maize." Plant biotechnology journal 15 (2):257-268.

Dasgupta, MK. 1992. Rice sheath blight: The challenge continues. Singh, US et al., (ed.). Plant Diseases of International Importance. Volume I. Diseases of Cereals and Pulses. Prentice Hall, Englewood CKffis, New Jersey.

Hiei, Yukoh, and Toshihiko Komari. 2008. "Agrobacterium-mediated transformation of rice using immature embryos or calli induced from mature seed." Nature protocols 3 (5):824.

Jiang, Fuguo, and Jennifer A Doudna. 2017. "CRISPR-Cas9 structures and mechanisms." Annual review of biophysics 46:505-529.

Kidd, Brendan N, Cameron I Edgar, Krish K Kumar, Elizabeth A Aitken, Peer M Schenk, John M Manners, and Kemal Kazan. 2009. "The mediator complex subunit PFT1 is a key regulator of jasmonate-dependent defense in Arabidopsis." The Plant Cell 21 (8):22372252.

Liu, Hao, Yuduan Ding, Yanqing Zhou, Wenqi Jin, Kabin Xie, and Ling-Ling Chen. 2017. "CRISPR-P 2.0: an improved CRISPRCas9 tool for genome editing in plants." Molecular plant 10 (3):530-532.

Ran, F Ann, Patrick D Hsu, Jason Wright, Vineeta
Agarwala, David A Scott, and Feng Zhang. 2013. "Genome engineering using the CRISPR-Cas9 system." Nature protocols 8 (11):2281.

Savary, Serge, Laetitia Willocquet, Francisco A Elazegui, Nancy P Castilla, and Paul S Teng. 2000. "Rice pest constraints in tropical Asia: quantification of yield losses due to rice pests in a range of production situations." Plant disease 84 (3):357-369.

Shan, Qiwei, Yanpeng Wang, Jun Li, and Caixia Gao. 2014. "Genome editing in rice and wheat using the CRISPR/Cas system." Nature protocols 9 (10):2395.

Sun, Yongwei, Xin Zhang, Chuanyin Wu, Yubing He, Youzhi Ma, Han Hou, Xiuping Guo, Wenming Du, Yunde Zhao, and Lanqin Xia. 2016. "Engineering herbicide-resistant rice plants through CRISPR/Cas9-mediated homologous recombination of acetolactate synthase." Molecular plant 9 (4):628-631.

Thatcher, Louise F, John M Manners, and Kemal Kazan. 2009. "Fusarium oxysporum hijacks COI1-mediated jasmonate signaling to promote disease development in Arabidopsis." The Plant Journal 58 (6):927-939.

Xie, Kabin, Bastian Minkenberg, and Yinong Yang. 2014. "Targeted gene mutation in rice using a CRISPR-Cas9 system." Bio Protoc 4:e1225.

Zong, Yuan, Yanpeng Wang, Chao Li, Rui Zhang, Kunling Chen, Yidong Ran, Jin-Long Qiu, Daowen Wang, and Caixia Gao. 2017. "Precise base editing in rice, wheat and maize with a Cas9-cytidine deaminase fusion." Nature biotechnology 35 (5):438.

\section{How to cite this article:}

Priya Rajesh Shah, S. Varanavasiappan, E. Kokiladevi, A. Ramanathan and Kumar, K.K. 2019. Genome Editing of Rice PFT1 Gene to Study its Role in Rice Sheath Blight Disease Resistance. Int.J.Curr.Microbiol.App.Sci. 8(06): 2356-2364.

doi: https://doi.org/10.20546/ijcmas.2019.806.281 